\title{
O estresse do estudante de Medicina durante a pandemia de COVID-19
}

\author{
Medical student stress during the COVID-19 pandemic \\ Estrés de estudantes Médicos durante la pandemia COVID-19
}

Recebido: 18/07/2021 | Revisado: 25/07/2021 | Aceito: 26/07/2021 | Publicado: 03/08/2021

Talita de Oliveira Felippe

ORCID: https://orcid.org/0000-0003-2143-8389

Universidade do Planalto Catarinense, Brasil

E-mail: talitafelippe@uniplaclages.edu.br

Claudia Marchezan Spaniol

ORCID: https://orcid.org/0000-0003-3792-295X

Universidade do Planalto Catarinense, Brasil

E-mail:claudiaspaniol@uniplaclages.edu.br

Lucas Auada da Silva

ORCID: https://orcid.org/0000-0001-7533-6666

Universidade do Planalto Catarinense, Brasil

E-mail: lucas_auada@hotmail.com

André Chaves Calabria

ORCID: https://orcid.org/0000-0001-6033-422X

Universidade do Planalto Catarinense, Brasil

E-mail: andre.calabria@hotmail.com

Gabrielle Ferreira

ORCID: https://orcid.org/0000-0002-1527-0252

Universidade do Planalto Catarinense, Brasil

E-mail: Gabrielleferreira1015@gmail.com

Nichollas de Lorenzi Carvalho

ORCID: https://orcid.org/0000-0002-5140-6479

Universidade do Planalto Catarinense, Brasil

E-mail: nlorenzicarvalho@gmail.com

Murilo Moretti

ORCID: https://orcid.org/0000-0002-9560-3415

Hospital Nossa Senhora da Conceição, Brasil E-mail: murilo_moretti@hotmail.com

Natalia Veronez da Cunha Bellinati

ORCID: https://orcid.org/0000-0002-8522-5836

Universidade do Planalto Catarinense, Brasil E-mail: nat_cunha@uniplaclages.edu.br

\begin{abstract}
Resumo
Objetivo: Avaliar o estresse do estudante de medicina frente a pandemia de COVID-19. Método: Estudo quantitativo, observacional, transversal. Participaram da pesquisa 320 alunos regularmente matriculados no curso de medicina de qualquer Instituição de Ensino Superior (IES) brasileira, do primeiro ao sexto ano, maiores de 18 anos. A coleta de dados foi realizada por meio virtual, com o envio de um link para acesso do instrumento de pesquisa autoaplicável, contendo questões sobre o perfil sociodemográfico e estilo de vida e a Escala de Estresse Percebido. A pesquisa foi aprovada pelo Comitê de Ética em Pesquisa da Universidade do Planalto Catarinense (número 072659/2020). Os dados foram então submetidos à análise estatística descritiva. Resultados: A pandemia impactou negativamente no estilo de vida dos estudantes, que diminuíram a prática de atividades físicas e pioram a alimentação. Ainda, 46,6\% dos estudantes apresentaram um agravo ou desenvolvimento do transtorno psicopatológico de ansiedade. Pela Escala de Estresse Percebido, observou-se que a grande maioria dos estudantes de medicina $(75,6 \%)$ apresentaram um nível de estresse médio (31 \pm 4$)$. Conclusão: A pandemia de COVID-19 impactou negativamente os estudantes de medicina, deixando-os mais ansiosos e estressados. Faz-se necessário implementação de redes de apoio à saúde mental nas universidades para esses estudantes. Além disso, também é preciso maior suporte ao estudante no retorno às atividades práticas, com fornecimento correto de todos EPIs e maiores capacitações para o enfrentamento a COVID19.
\end{abstract}

Palavras-chave: COVID-19; Acadêmicos de medicina; Estresse; Saúde mental.

\footnotetext{
Abstract

Objective: Evaluate the stress of medical students facing the COVID-19 pandemic. Method: Quantitative, observational, cross-sectional study. 320 students regularly enrolled in the medical course of any Brazilian Higher Education Institution (HEI) participated in the research, from the first to sixth year, in legal age. Data collection was
} 
carried out online, with the sending of a link to access the self-administered survey instrument, containing questions about the sociodemographic profile and lifestyle and the Perceived Stress Scale. The research was approved by the Research Ethics Committee of the Universidade do Planalto Catarinense (number 072659/2020). Data were then submitted to descriptive statistical analysis. Results: The pandemic negatively impacted the lifestyle of students, who reduced the practice of physical activities and worsened their diet. Also, 46.6\% of the students had a problem or development of psychopathological anxiety disorder. Through the Perceived Stress Scale, it was observed that the vast majority of medical students $(75.6 \%)$ had an average stress level (31 \pm 4$)$. Conclusion: The COVID-19 pandemic impacted negatively medical students, making them more anxious and stressed. It is necessary to implement mental health support networks in universities for these students. In addition, greater support is also needed for the student in returning to practical activities, with the correct supply of all PPE and greater training to face COVID-19.

Keywords: COVID-19; Medical students; Stress; Mental health.

\section{Resumen}

Objetivo: Evaluar lo estrés de los estudiantes de medicina que enfrentan la pandemia de COVID-19. Método: Estudio cuantitativo, observacional, transversal. Participaron de la investigación 320 estudiantes matriculados regularmente en el curso de medicina de la Institución de Educación Superior (IES) brasileña, desde el primero hasta lo sexto año, mayores de 18 años. La recolección de datos se realizó en línea, con el envío de un enlace para acceder lo instrumento de encuesta autoadministrado, que contiene preguntas sobre el perfil sociodemográfico y estilo de vida y la Escala de Estrés Percibido. La investigación fue aprobada por el Comité de Ética en una Investigación de la Universidade do Planalto Catarinense (n. 072659/2020). Luego, los datos se sometieron a análisis estadística descriptiva. Resultados: La pandemia tuvo un impacto negativo en el estilo de vida de los estudiantes, lo que redujo la práctica de actividades físicas y empeoró su dieta. Además, el 46,6\% de los estudiantes tuvo algún problema o desarrollo de trastorno de ansiedad psicopatológica. A través de la Escala de Estrés Percibido, se observó que la gran mayoría de los estudiantes de medicina $(75,6 \%)$ tenían un nivel de estrés medio $(31 \pm 4)$. Conclusión: La pandemia de COVID-19 afectó negativamente a los estudiantes de medicina, haciéndolos más ansiosos y estresados. Es necesario implementar redes de apoyo a la salud mental en las universidades para estos estudiantes. Además, también se necesita un mayor apoyo para que el alumno vuelva a las actividades prácticas, con el correcto suministro de todos los EPI y también una mayor formación para afrontar el COVID-19.

Palabras clave: COVID-19; Médicos académicos; Estrés; Salud mental.

\section{Introdução}

A pandemia de COVID-19 fez-se rapidamente um risco para os sistemas de saúde do mundo inteiro. As principais medidas tomadas pelos governos estabeleceram contramedidas para conter os efeitos da doença, sendo a prevenção com objetivo de reduzir a transmissão comunitária uma das medidas mais eficazes (Cascella et al., 2020). As medidas não farmacológicas têm o poder de reduzirem o número de casos e o impacto para os serviços de saúde, já que reduzem o pico epidêmico, permitindo uma distribuição dos casos ao longo do tempo e um não esgotamento dos serviços de saúde (Brasil, 2020b). Através do reconhecimento da Organização Mundial de Saúde e com o apoio científico de diversos outros importantes órgãos, como a Sociedade Brasileira para o Progresso da Ciência, Sociedade Brasileira de Infectologia e Academia Brasileira de Ciências, o Ministério da Saúde atualizou definições operacionais e recomendou às Secretarias de Saúde dos Municípios, Estados e Distrito Federal para avaliarem a adoção de medidas preventivas e isolamento social da população (Brasil, 2020a). Mediante a emergência da situação, governos estaduais e municipais implementaram o período de quarentena até mesmo para indivíduos suscetíveis sem histórico de contato, por período indeterminado, como medida profilática, com o fechamento de escolas, universidades, bares e boates, interrupção de eventos coletivos e isolamento vertical das indústrias. Segundo o Imperial College de Londres, o número de óbitos poderia chegar até 1,15 milhão caso não ocorresse o isolamento no Brasil (Rodriguez-Morales et al., 2020).

O termo quarentena é a separação e restrição de movimento de pessoas que possuem alto potencial de exposição para uma doença contagiosa, para que seja reduzido o risco de infectar outros indivíduos. Indica que deve ser mantido o isolamento, com restrição à circulação. Essas medidas já foram tomadas anteriormente para o controle de doenças como hanseníase, peste negra, SARS, ebola e influenza H1N1 (Webster et al., 2020). Diversos fatores estressores que podem gerar danos psicológicos durante a quarentena, sendo os principais o a modificação da rotina; limitação da mobilidade; afastamento das pessoas; medo 
de infecção; frustração, tédio, suprimentos inadequados e perdas financeiras (Brooks et al., 2020). Ainda, segundo Brooks et al. (2020), os indivíduos podem desenvolver sintomas de estresse pós-traumático, depressão, abuso de substâncias, estado confusional e irritabilidade.

Nos profissionais de saúde, observou-se maior chance de os fatores de estresse os atingirem, assim como maior medo de autoinfecção e contaminação de seus familiares. Dessa forma, a probabilidade de ocorrência de exaustão, ansiedade, distanciamento social e relutância ao trabalho é maior (Brooks et al., 2020). Além disso, nesse período de pandemia, os acadêmicos de medicina também podem ter uma maior probabilidade de sofrer estresse e danos psicológicos, já que os principais fatores de estresse desses estudantes são ampliados, como o contato com a morte e sofrimento, desconstrução da onipotência médica, consciência dos problemas da profissão, medo de adquirir doenças, medo de transmitir doença aos familiares, insegurança com o cumprimento do ano letivo, entre outros (Barbosa et al., 2015; Moreira, Vasconcellos, \& Heath, 2015; Santa, \& Cantilino, 2016; Kaluf et al., 2019). Todos esses fatores, juntamente com o período de quarentena podem levar ao aumento do estresse, já que em contexto não pandêmico a população de acadêmicos de medicina apresenta o maior número de suicídios e transtornos psicopatológicos como Transtorno Depressivo Maior (TDM) e Transtorno de Ansiedade Social (TAS) (Santa \& Cantilino, 2016; Trindade, Sousa \& Carreira, 2021).

Assim, diante desse contexto, o presente trabalho tem por objetivo avaliar o estresse do estudante de medicina frente a pandemia de COVID-19.

\section{Metodologia}

Trata-se de estudo quantitativo (Estrela, 2018), observacional, transversal, previamente aprovada pelo Comitê de Ética em Pesquisa da Universidade do Planalto Catarinense (número 072659/2020).

Participaram do estudo 320 alunos regularmente matriculados no curso de medicina de qualquer Instituição de Ensino Superior (IES) brasileira, do primeiro ao sexto ano, maiores de 18 anos.

A pesquisa foi realizada de maneira virtual, por meio do envio de um link para acesso do instrumento de pesquisa autoaplicável. Este era constituído de duas partes: a primeira, desenvolvida pelas pesquisadoras, com base na literatura, visando conhecer o perfil sociodemográfico, acadêmico e estilo de vida dos estudantes. Estruturado com questões sobre sexo, idade, Estado em que reside, universidade que está matriculado, qual fase do curso de medicina está cursando, como foram mantidas as atividades acadêmicas durante o período de quarentena e qual a satisfação do estudante com as medidas tomadas. Sobre seus hábitos de vida, foi questionado se o estudante iniciou ou aumentou o uso de substâncias psicoativas durante o período de quarentena, se realizavam atividades físicas regulares e como manteve sua alimentação.

Já a segunda parte consistiu na Escala de Estresse Percebido, validada por Luft et al. (2007), a qual faz 14 questionamentos sobre a frequência de como a pessoa se sentiu durante o último mês. Os itens foram designados para verificar o quanto imprevisível, incontrolável e sobrecarregada os participantes avaliam sua vida. O elevado nível de estresse percebido, mensurado pela escala, está diretamente associado com desequilíbrios fisiológicos, como altos níveis de cortisol, triglicérides, interleucina-6 (IL-6), entre outros (Luft et al., 2007). O escore final pode variar de zero a 54, sendo que valores maiores representam maior estresse. Para interpretação, foi realizada a estratificação do escore de estresse em elevado, regular e baixo, assumindo-se a média obtida $(30,76 \pm 4,11)$ como ponto de referência. A distância entre os desvios padrão e a média constituiu o nível regular de estresse, e os escores abaixo e acima desses valores foram considerados como níveis baixos e elevados. A estratificação obtida está demonstrada na Tabela 1. 
Tabela 1- Estratificação do escore de estresse.

\begin{tabular}{ll}
\hline Escore de Estresse & \\
\hline Estresse elevado & $\geq 26$ \\
Estresse regular & 27 a 35 \\
Estresse baixo & $\leq 36$ \\
\hline
\end{tabular}

Fonte: Própria (2021).

Os dados foram então tabulados no programa Excel® Microsoft 2008 e exportados para o SestatNet. Após isso, submetidos à análise estatística descritiva (média, desvio padrão e frequência).

\section{Resultados}

Os estudantes de medicina participantes da pesquisa têm entre 18 e 50 anos, com idade média de $23 \pm 4$ anos. A análise dos dados sociodemográficos revelou que $73,1 \%$ dos participantes são do gênero feminino. No que se refere a universidade que frequenta, 70,9\% são de IES privadas. Os estudantes que responderam à pesquisa são de 75 universidades diferentes do Brasil, provenientes de 16 estados. Houve participação de acadêmicos de todas as fases, com maior prevalência da sexta (22,8\%). Durante a aplicação do instrumento, 89,7\% dos participantes ficaram com aulas/tutorias online e avaliações, sendo que, $47,5 \%$ não ficaram satisfeitos com as metodologias usadas pela universidade durante o período de isolamento. Sobre a insegurança/preocupação com o cumprimento do cronograma letivo, 93,1\% responderam que ficaram inseguros e preocupados. Dos participantes da pesquisa, apenas $24,1 \%$ se inscreveram para a ação governamental "O Brasil conta comigo!’. Após o retorno das atividades ou ação voluntária, 56,6\% tiveram medo de se contaminar e 67,8\% ficaram com medo de contaminar familiares ou pessoas próximas (Tabela 1).

Tabela 1 - Características sociodemográficas e de atividades acadêmicas dos participantes (n=320).

\begin{tabular}{|c|c|c|c|}
\hline Variável & Categoria & $\mathbf{n}$ & $\%$ \\
\hline \multicolumn{4}{|l|}{ Gênero } \\
\hline & Feminino & 23 & 73,1 \\
\hline & Masculino & 86 & 26,9 \\
\hline \multicolumn{4}{|l|}{ Universidade } \\
\hline & Privada & 22 & 70,9 \\
\hline & Comunitária & -50 & 15,6 \\
\hline & Pública & 43 & 13,4 \\
\hline \multicolumn{4}{|l|}{ Fase do curso } \\
\hline & $1^{\mathrm{a}}$ & 03 & 0,9 \\
\hline & $2^{a}$ & 30 & 9,4 \\
\hline & $3^{a}$ & 9 & 2,8 \\
\hline & $4^{\mathrm{a}}$ & 40 & 12,5 \\
\hline & $5^{\mathrm{a}}$ & 21 & 6,6 \\
\hline & $6^{\mathrm{a}}$ & 73 & 22,8 \\
\hline & $7^{\mathrm{a}}$ & 51 & 15,9 \\
\hline & $8^{\mathrm{a}}$ & 45 & 14,1 \\
\hline & $9^{a}$ & 18 & 5,6 \\
\hline & $10^{\mathrm{a}}$ & 21 & 6,6 \\
\hline & $11^{\mathrm{a}}$ & 4 & 1,3 \\
\hline & $12^{\mathrm{a}}$ & 5 & 1,6 \\
\hline
\end{tabular}




$\begin{array}{lllr}\begin{array}{l}\text { Atividades acadêmicas durante a } \\ \text { quarentena }\end{array} & \begin{array}{l}\text { Com aulas/tutorias online e } \\ \text { avaliações }\end{array} & 28 & 89,7 \\ & \begin{array}{l}\text { Com aulas/tutorias online e sem } \\ \text { avaliações }\end{array} & 14 & 4,4 \\ & \text { Atividades totalmente paradas } & 19 & 5,9\end{array}$

\title{
Satisfação com metodologias adotadas durante a quarentena
}

\begin{tabular}{llrr} 
& Satisfeitos & 16 & 52,5 \\
& Insatisfeitos & 15 & 47,5 \\
\hline
\end{tabular}

Insegurança/Preocupação com cumprimento do cronograma letivo

$\begin{array}{lcc}\text { Sim } & 29 & 93,1 \\ \text { Não } & 22 & 6,9\end{array}$

\begin{abstract}
Inscrição voluntária para a ação governamental "O Brasil Conta Comigo!”
\end{abstract}

$\begin{array}{lll}\text { Sim } & 77 & 24,1 \\ \text { Não } & 24 & 75,9\end{array}$

Medo de se contaminar após o retorno

das atividades práticas

$\begin{array}{lll}\text { Sim } & 18 & 56,6 \\ \text { Não } & 74 & 23,1 \\ \text { Não retornou às atividades } & 65 & 20,3\end{array}$

\begin{tabular}{llll}
\hline $\begin{array}{l}\text { Medo de contaminar seus familiares ou } \\
\text { pessoas próximas }\end{array}$ & & & \\
& Sim & 21 & 67,8 \\
& Não & 35 & 10,9 \\
& Não retornou às atividades & 68 & 21,3 \\
\hline
\end{tabular}

Fonte: Própria (2021).

$\mathrm{Na}$ avaliação do estilo de vida, evidenciou-se uma piora da mesma, com a diminuição da prática de atividades físicas, piora da alimentação e aumento do consumo de industrializados e carboidratos.

Antes da pandemia 61,6\% dos entrevistados praticavam atividades físicas. Já durante o período de isolamento, esse percentual reduziu para 45,3\%. Sobre a alimentação dos acadêmicos, antes da pandemia 32,5\% dos entrevistados responderam possuir uma alimentação saudável, 57,2\% moderada e 10,3\% não saudável. Já durante o período de isolamento, 25,3\% dos estudantes relataram ter uma alimentação saudável, 54,1\% moderada e 20,6\% não saudável.

Além disso, a saúde mental dos acadêmicos também piorou, já que 46,6\% relataram ter um agravo ou desenvolvimento do transtorno psicopatológico de ansiedade. Por fim, 21,1\% iniciaram ou aumento o consumo de substâncias psicoativas (álcool, drogas, tabagismo - Tabela 2). 
Tabela 2- Estilo de vida dos participantes $(n=320)$.

\begin{tabular}{|c|c|c|c|}
\hline Variável & Categoria & $\mathbf{n}$ & $\%$ \\
\hline \multicolumn{4}{|c|}{$\begin{array}{l}\text { Estado de humor mais prevalente durante o } \\
\text { isolamento }\end{array}$} \\
\hline & Ansiedade & 101 & 31,6 \\
\hline & Preocupação e insegurança & 98 & 30,6 \\
\hline & Tédio & 47 & 14,7 \\
\hline & Alegria & 17 & 5,3 \\
\hline & Tristeza & 33 & 10,3 \\
\hline & Raiva & 09 & 2,8 \\
\hline & Outros & 15 & 4,7 \\
\hline
\end{tabular}

Desenvolveram ou tiveram algum agravo de transtorno psicopatológico durante a quarentena

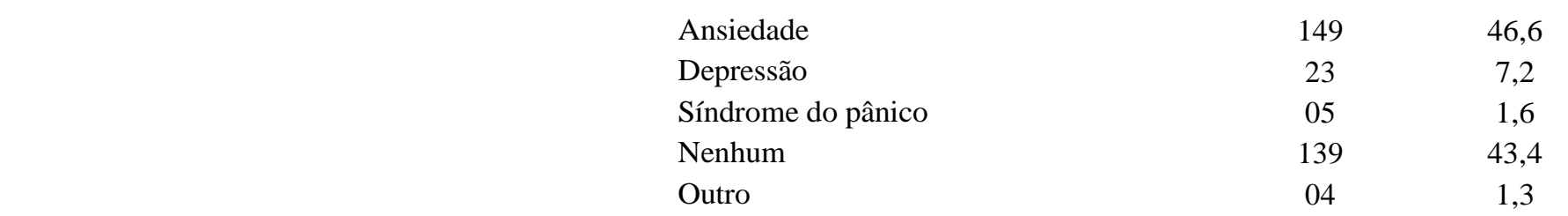

Realizavam atividades físicas regulares antes da quarentena

\begin{tabular}{llrr} 
& Sim & 197 & 61,6 \\
& Não & 123 & 38,4 \\
\hline
\end{tabular}

\section{Realizavam atividades físicas regulares durante} da quarentena

\begin{tabular}{|c|c|c|c|}
\hline & Sim & 145 & 45,3 \\
\hline & Não & 175 & 54,7 \\
\hline \multicolumn{4}{|l|}{ Alimentação antes da quarentena } \\
\hline & Saudável & 104 & 32,5 \\
\hline & Moderada & 183 & 57,3 \\
\hline & Não saudável & 33 & 10,3 \\
\hline \multicolumn{4}{|l|}{ Alimentação durante a quarentena } \\
\hline & Saudável & 81 & 25,3 \\
\hline & Moderada & 173 & 54,1 \\
\hline & Não saudável & 66 & 20,6 \\
\hline \multicolumn{4}{|c|}{$\begin{array}{l}\text { Iniciou ou aumentou o consumo de substâncias } \\
\text { psicoativas durante a quarentena }\end{array}$} \\
\hline & Sim & 77 & 24,1 \\
\hline & Não & 243 & 75,9 \\
\hline
\end{tabular}

Fonte: Própria (2021).

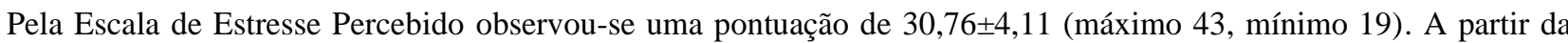
estratificação, nota-se que 75,6\% dos estudantes apresentavam estresse médio, 13,1\% estresse baixo e 11,3\% estresse alto.

Do total de estudantes entrevistados, aqueles que estavam com as aulas práticas presenciais suspensas no momento da entrevista $(65-20,3 \%), 16,9 \%$ apresentaram níveis de estresse menores, 78,5\% médio e apenas 3 (4,6\%) apresentaram níveis de estresse elevado. Em contrapartida, os estudantes que já haviam retornado as práticas presenciais (79,6\%), 12,94\% apresentaram nível de estresse elevado. 
Dos 149 acadêmicos entrevistados (46,6\%) que relataram desenvolver ou agravar o quadro pré-existente de ansiedade, $138(92,6 \%)$ apresentaram nível de estresse médio ou alto, indicando uma possível relação entre ansiedade e estresse.

\section{Discussão}

Os achados sociodemográficos do estudo demonstraram que a maioria dos acadêmicos de medicina atualmente são do gênero feminino e estudam em universidades privadas. A maioria dos estudantes ficaram com aulas/tutorias online e avaliações durante o isolamento social devido a pandemia, quase metade dos entrevistados estavam insatisfeitos com a metodologia adotada e mais de $90 \%$ estavam inseguros/preocupados com o cumprimento do ano letivo. A maioria dos entrevistados demonstraram medo de se contaminar ao retornar para as atividades práticas, os quais apontaram medo ainda maior em contaminar familiares ou pessoas próximas. Em relação a saúde mental e estilo de vida, o estado de humor e transtorno psicopatológico mais agravado/gerado durante esse período foi de ansiedade. A maioria dos estudantes reduziram a prática de atividade física e passaram a ter uma alimentação não saudável. Ainda, uma parcela considerável dos mesmos iniciou ou agravou o uso de substâncias psicoativas durante esse mesmo período.

Nas últimas décadas, organismos como a Organização Mundial de Saúde (OMS), a Organização Pan-Americana de Saúde (OPAS) e a Associação Brasileira de Educação Médica (Abem) têm manifestado grande preocupação com o ensino médico. Nesse contexto, um maior conhecimento do perfil sociodemográfico e socioeconômico dos alunos é de extrema importância (Cardoso et al., 2015). Em geral, os cursos de Medicina apresentaram por muitos anos um perfil predominante de estudantes: sexo masculino, de classes sociais mais elevadas, provenientes de grandes centros urbanos. Todavia, atualmente percebe-se uma mudança neste perfil, tanto nos entrevistados da pesquisa quanto nos estudos recentes, relacionadas a mudanças nos processos de admissão, como ações afirmativas e sistemas de cotas, visando ampliar o acesso às instituições públicas de ensino superior (Souza et al., 2020). Além disso, nota-se um processo de aumento marcante do sexo feminino na profissão médica, quanto nas faculdades de medicina, conforme o resultado da pesquisa. Os homens ainda eram maioria entre os médicos em atividade em 2017, contudo, as mulheres já eram maioria entre os médicos mais jovens - representavam 57,4\% no grupo até 29 anos e 53,7\% na faixa entre 30 e 34 anos (Do Rego et al., 2019).

A maioria das escolas médicas no Brasil são privadas, seguidas de instituições públicas e comunitárias respectivamente, indo de acordo com os resultados da presente pesquisa. Isso demonstra que, apesar das políticas de ação afirmativa, o curso de medicina continua sendo de elite (Veras et al., 2020).

Quando os participantes foram indagados sobre o estilo de vida após o início da pandemia de COVID-19 a maioria relatou reduzir a prática de atividade física e ingerir mais alimentos ultra processados, ricos em gorduras saturadas e açucares. Além disso, uma parcela considerável iniciou ou aumentou o uso de substâncias psicoativas. Segundo a World Health Organization (WHO, 1998), o estilo de vida do indivíduo é considerado um importante moderador de saúde, sendo definido por um conjunto identificável de hábitos e costumes influenciados principalmente pelos contextos sociais, econômicos e ambientais. Dentre os padrões comportamentais do estilo de vida, a World Health Organization (2020) destaca o uso de tabaco e álcool, a atividade física e a dieta. Apesar de a alimentação saudável e prática de atividades físicas serem os principais pilares de um bom estilo de vida e consequentemente prevenção de doenças crônicas, diversos estudos sobre o assunto revelam que os estudantes de medicina possuem comportamentos de risco para a saúde, com decréscimo da qualidade de vida ao longo do curso (Loureiro, 2006). A faculdade de medicina, mentalmente desafiadora, com elevada carga horária, grande quantidade de assuntos e várias atividades práticas em hospitais e unidades de saúde, leva o aluno a abdicar da prática de exercícios físicos e optar por uma alimentação rica em industrializados e pobre em nutrientes, extremamente comum em um estilo de vida apressado e estressante (Barbosa et al., 2015). 
Em estudo realizado por Bosi et al. (2014), com 200 acadêmicas de medicina da Universidade Federal do Rio de Janeiro (UFRJ), 45,5\% das alunas de medicina entrevistadas tiveram comportamentos alimentares anormais ou de risco para desenvolvimento de Transtorno Compulsivo Alimentar (TCA). Além disso, há um aumento da prevalência de Hipertensão Arterial (HAS) e dislipidemia ao longo do curso de medicina (Barbosa et al., 2015). Diante desses dados temos uma grave problemática, já que esses são indivíduos que estão sendo formados para cuidar da saúde da população, sendo necessárias medidas de intervenção no transcorrer do curso que melhorem a compreensão e adesão dos acadêmicos de boas práticas de saúde (Neto et al., 2013).

É evidente que a pandemia impactou diretamente na diminuição do convívio social, com restrições advindas do distanciamento social e impossibilidade de uso dos espaços públicos de lazeres ativos, fechamento de academias, clubes e parques. O envolvimento em experiências de lazer diminuiu, na pandemia, para todos os grupos etários, entretanto no contexto dos universitários o distanciamento social aumentou, com a "residencialização" e "virtualização" do lazer, reduziu as atividades físicas no lazer, com aumento do comportamento sedentário, devido maior demanda de atividades online (Tavares et al., 2020).

As implicações acerca da diminuição na prática de atividade física durante a pandemia de COVID-19 podem ser diversas. Segundo Marin et al. (2021), o maior sedentarismo, a redução da prática de exercício físico, tempo de sono e piora da dieta foram fatores de auxílio no desenvolvimento de Transtorno Depressivo Maior (TDM) em universitários durante a pandemia (Marin et al., 2021). Ademais, um pior estilo de vida pode favorecer o surgimento de Doenças Crônicas Não Transmissíveis (DCNT), as quais são patologias multifatoriais de longa duração, impulsionadas principalmente pelo estilo de vida não saudável, como dieta inadequada, inatividade física e uso de tabaco e álcool. Dentre as principais DCNT, estão a doença cardiovascular, doença respiratória crônica, diabetes e câncer (World Health Organization, 2020). As DCNT compõem uma epidemia vigente, sendo a causa principal de mortalidade e de incapacidade prematura na maioria dos países do continente americano, incluindo o Brasil (Organização Pan Americana de Saúde, 2018). Ainda, um estilo de vida não saudável leva a um aumento de peso corporal e diminuição dos níveis de imunidade fisiológica (Tavares et al., 2020).

Em um cenário de pandemia, é ainda mais importante ter um estilo de vida saudável, sendo uma importante ferramenta para a saúde pública no enfrentamento da COVID-19. Além disso, a prática de atividade física permite redefinir um estado de equilíbrio, em relação às necessidades biopsicossociais do ser humano. Também, possui extrema importância na prevenção de doenças mentais e DCNT, mantendo o bem-estar em tempos tão difíceis e adversos (Tavares et al., 2020).

Foi relevante também o resultado de que os estudantes entrevistados que estavam com aulas práticas presenciais suspensas durante o momento da entrevista, apresentaram níveis de estresse menores. A maioria dos alunos que retornaram às atividades práticas presenciais relataram medo frequente de se contaminar com o vírus, mesmo com os devidos cuidados. Diversos fatores podem estar relacionados a esse medo de contaminação, mesmo com uso de EPI's e medidas preventivas, como incertezas e desconhecimento em torno do patógeno, urgência por respostas, informações dúbias ou falsas sobre o vírus, medidas de controle insuficientes, falta de mecanismos terapêuticos eficazes e crescimento exponencial no número de casos (Ornell Felipe et al., 2020; Freitas et al., 2021). A adoção de medidas não-farmacológicas para controlar a propagação da infecção, tendo como exemplo a higienização das mãos - por meio da lavagem com água e sabão ou antissepsia utilizando gel à base de álcool 70\% - se faz necessária em decorrência da falta de medicamentos específicos e vacinas no momento da entrevista (de Souza Neto \& de Freitas, 2020).

Apesar de essencial, sabe-se também que muitos locais enfrentam a falta de EPI's e boas condições de trabalho/estágio, o que se caracteriza como um grande fator gerador de medo e estresse nos profissionais de saúde e acadêmicos de medicina. Segundo levantamento de abril de 2020 da Associação Paulista de Medicina (APM), pelo menos metade dos médicos sofrem com falta de equipamentos de proteção individual (EPIs), como máscaras N95, em seus locais de 
trabalho, pondo em risco a própria segurança para o enfrentamento da pandemia (Freitas et al., 2021) Além disso, a realidade hospitalar atual não segue mais a rotina da graduação como antigamente com suspensão de consultas ambulatoriais nos setores de clínica médica, pediatria e saúde coletiva, prejudicando o processo de ensino-aprendizagem e causando ainda mais insegurança e preocupação nos estudantes, grande fator gerador de estresse (Freitas et al., 2021). De fato, a literatura e o resultado da pesquisa apontam que o medo de infecção é um importante agente estressor, com potencial para causar danos psicológicos significativos, incluindo depressão, irritabilidade, estado confusional e abuso de substâncias (Brooks et al., 2020). Esses danos psicológicos e abuso de substâncias também puderam ser encontrados na pesquisa, visto que $24,1 \%$ iniciaram/aumentaram o uso de substâncias psicoativas. Além disso, houve uma piora na saúde mental dos estudantes, já que a maioria relatou prevalência de estados de humor como ansiedade, preocupação e tristeza, além de 46,6\% terem relatado um agravo ou desenvolvimento do transtorno psicopatológico de ansiedade. Os transtornos mentais comuns (TMC) geram sofrimento psíquico, interferindo nos relacionamentos interpessoais, atividades diárias e qualidade de vida. Estima-se que os TMC atinjam de $9 \%$ a $12 \%$ da população mundial e de 12 a 15\% da brasileira em todas as faixas etárias (Sacramento et al., 2021). Dentre os diferentes grupos sociais, os estudantes de medicina possuem maior vulnerabilidade para desenvolver transtornos de ansiedade e depressão, assim como ideação suicida (Soeiro et al., 2021). A exaustiva rotina de estudos, experiências de contato com doenças e com a morte podem ser os possíveis potencializadores para o desencadeamento de transtornos psicopatológicos nesses acadêmicos (Soeiro et al., 2021; Trindade, Sousa \& Carreira, 2021). Em estudo realizado no curso de medicina em uma faculdade de Salvador-BA, constatou-se a prevalência de sintomas de 30,8\% para ansiedade e $36 \%$ para depressão (Sacramento et al., 2021).

Na pandemia o número de pessoas cuja saúde mental é afetada tende a ser maior que o número de pessoas afetadas pela infecção. Esse comprometimento da saúde mental pode trazer implicações que podem durar mais tempo e ter maior prevalência que a própria epidemia, como já foi demonstrado em outras períodos de comprometimento da saúde coletiva (Ornell Felipe et al., 2020). Esses dados são de extrema preocupação, tendo em vista que de acordo com os resultados da pesquisa, a maioria dos estudantes tiveram uma piora da saúde mental, o que pode gerar dados ainda piores em relação a prevalência de transtornos psicopatológicos nos acadêmicos de medicina. Segundo Brooks et al. (2020), os prejuízos psicopatológicos que podem ser gerados nesse período, principalmente nos profissionais de saúde, são sintomas de Transtorno de Ansiedade Generalizada (TAG) com crises de pânico, Transtorno de Estresse Pós-Traumático (TEPT), depressão, abuso de substâncias, estado confusional e irritabilidade. Ainda, pessoas que já possuem histórico de transtornos mentais têm maior predisposição de desenvolver danos psicológicos nesse tempo e após (Brooks et al., 2020; Santa \& Cantilino, 2016)

Segundo Freitas et al. (2021), embora alguns protocolos médicos foram estabelecidos a maioria dos profissionais da saúde não é treinada para prestar assistência em saúde mental durante a pandemia, assim como não recebe atendimento especializado. Destacando-se altas taxas de ansiedade, estresse e transtornos mentais nessa população durante o enfrentamento de pandemias. Todos esses fatores evidenciam a necessidade do aumento de pesquisas e desenvolvimento de protocolos efetivos para maior cuidado da saúde mental dos profissionais da saúde em paralelo a surtos infecciosos (Ornell Felipe et al., 2020).

Conforme resultado da pesquisa, observou-se um nível elevado de estudantes com nível moderado de estresse. O estresse pode ser entendido como conjunto de estímulos que prejudicam a homeostasia do corpo frente a situações notadas como ameaças. Além de estar associado a diversas queixas psicopatológicas e a outras doenças como HAS, gastrite e câncer. Pode também afetar a qualidade de vida do indivíduo, gerando perda da qualidade do sono, irritabilidade, dificuldade de concentração e impaciência (Amorim et al., 2018). 
Estudantes de medicina estão expostos durante sua formação acadêmica, e até mesmo depois, a diversas situações geradoras de estresse. O despreparo para lidar com estas situações pode trazer importantes repercussões no desempenho acadêmico, na saúde e no bem-estar psicossocial desse estudante (de Sousa Furtado, de Oliveira Falcone \& Clark, 2003).

Os principais fatores desencadeadores de estresse nos acadêmicos de medicina são: a falta de tempo para o lazer e contato com amigos e familiares, grande quantidade de informações, contato com a morte e sofrimento, desconstrução da onipotência médica, consciência dos problemas da profissão, medo de adquirir doenças, dependência financeira em uma idade avançada, entre outros (Barbosa et al., 2015; Moreira, Vasconcellos, \& Heath, 2015; Santa, \& Cantilino, 2016; Kaluf et al., 2019). Todos esses fatores levam ao estresse e fazem com que os estudantes de medicina tenham as maiores taxas de suicídio, transtorno de ansiedade social (TAS), depressão, abuso de álcool e outras substâncias (Santa \& Cantilino, 2016; Trindade, Sousa \& Carreira, 2021). Um estudo realizado na Universidade Católica de Pernambuco com 249 alunos de medicina, mostrou que $72 \%$ possuíam má qualidade de sono e $66,9 \%$ transtornos psicopatológicos, sendo a prevalência de depressão de 18,6\% a 79\% nesses estudantes (Amorim et al., 2018).

Segundo Barbosa et al. (2015), em sua pesquisa feita com 482 estudantes da Escola Superior de Ciências da Santa Casa de Misericórdia de Vitória, o ingresso no mundo acadêmico levou muitos estudantes a iniciarem hábitos não saudáveis como ingestão de bebidas alcoólicas, tabagismo, sedentarismo e piora dos hábitos alimentares. Sendo as bebidas alcoólicas consideradas endêmicas no curso de medicina, visto que estudo já aponta um índice de 69,1\% dos estudantes fazendo uso de álcool pelo menos 1x na semana (Amorim et al., 2018). Outras substâncias psicoativas também são usadas como forma de alívio de estresse pelos estudantes, sendo o lança-perfume o segundo mais utilizado depois do álcool, cerca de 46,2\%, com a quantidade de consumo aumentada, progressivamente, ao longo do curso. (Lemos et al., 2007). Esse fato, de acordo com o resultado da presente pesquisa, piorou ainda mais, já que 24,1\% iniciaram ou aumentaram o uso de substâncias psicoativas durante a pandemia.

Segundo Brooks et al. (2020), durante o período de pandemia os profissionais de saúde estão ainda mais sujeitos ao estresse e danos psicológicos, com ocorrência de exaustão, ansiedade, distanciamento social e relutância ao trabalho maior. Isso ocorre devido a ampliação dos fatores de estresse, já que estão sujeitos a um maior isolamento social, ansiedade frente aos pacientes, resignação ao trabalho e prejuízo na performance laboral (Brooks et al., 2020). Aliado a isso, ainda, o medo de autoinfecção e contaminação de seus familiares e pessoas próximas, o qual foi relatado pela maioria dos estudantes da pesquisa. Os estudantes de medicina são atingidos por esses mesmos fatores de estresse dos profissionais da saúde, tendo ainda outras fontes de estresse que são amplificadas como o contato com a morte e sofrimento, desconstrução da onipotência médica, consciência dos problemas da profissão, medo de adquirir doenças, medo de transmitir doença aos familiares, insegurança com o cumprimento do ano letivo, entre outros (Barbosa et al., 2015; Moreira, Vasconcellos, \& Heath, 2015; Santa, \& Cantilino, 2016; Kaluf et al., 2019).

Além de todos os danos que o estresse pode trazer para os alunos, pode acarretar também em prejuízos para os pacientes, já que os estudantes estressados costumam perder a empatia e humanismo, estarem mais propensos a erros e com possível diminuição do desempenho acadêmico (Lima et al., 2016).

Apesar da existência de diversos estudos e do aumento do estresse dos acadêmicos durante a pandemia, a problemática do estresse nos estudantes de medicina continua sendo tabu, visto que a imagem social de equilíbrio e apoio que os estudantes têm de passar dificulta muito o pedido de auxílio. O aspecto emocional, muitas vezes, é negligenciado na formação do estudante de Medicina e falta nas universidades núcleos de apoio e matérias que ajudem nas habilidades emocionais, pois a formação acadêmica ainda persiste muito voltada apenas para o desempenho intelectual (Zonta, Robles \& Grosseman, 2006; Santa \& Cantilino, 2016). 


\section{Conclusão}

O presente estudo evidenciou a predominância de um estresse moderado nos estudantes de medicina durante a pandemia de COVID-19, com uma piora na qualidade de vida. Além disso, evidencia-se o aumento da do transtorno de ansiedade nesses estudantes, os quais em períodos pré-pandemia já possuíam dados preocupantes em relação a transtornos psicopatológicos. Diante desses fatos e do estresse que apesar de na grande maioria dos entrevistados ter sido moderado, devese ressaltar que esse período de isolamento pode ter repercussões inquantificáveis tanto para esses indivíduos quanto para seus futuros pacientes e comunidades. Fica evidente que é necessário maiores redes de apoio à saúde mental nas universidades para esses estudantes. Além disso, também é preciso maior suporte ao estudante no retorno às atividades práticas, com fornecimento correto de todos EPIs e maiores capacitações para o enfrentamento a COVID-19.

Novos estudos sobre a temática se fazem necessários, tais como pesquisas no campo de saúde mental dos estudantes de medicina no retorno às atividades práticas após o isolamento social e consequências da pandemia de COVID-19 para a formação desses acadêmicos.

\section{Agradecimentos}

Agradecemos ao Programa de Bolsas Universitárias de Santa Catarina - UNIEDU Art. 170/CE e Art. 171 pela bolsa de estudo da primeira autora.

\section{Referências}

Amorim, B. B., Moraes, L., Sá, I. C. G., Silva, B. B. G., \& Camara Filho, J. W. S. (2018). Saúde mental do estudante de medicina: psicopatologia, estresse, sono e qualidade de vida. Revista Psicologia, Diversidade e Saúde, 7(2), 245-254.

Barbosa, R. R., Martins, M. C. G., Carmo, F. P. T., Jacques, T. M., Serpa, R. G., Calil, A. O., \& Barbosa, L. F. M. (2015). Estudo sobre estilos de vida e níveis de estresse em estudantes de medicina. Int J Cardiovasc Sci, 28(4), 313-9.

Brooks, S. K., Webster, R. K., Smith, L. E., Woodland, L., Wesley, S., Greenberg, N., \& Rubin, G. J. (2020). The psychological impact of quarantine and how to reduce it: rapid review of the evidence. The lancet, 395(10227), 912-920.

Cardoso, F. D. A. B., Magalhães, J. F., Silva, K. M. L. D., \& Pereira, I. S. D. S. D. (2015). Perfil do estudante de Medicina da Universidade do Estado do Rio Grande do Norte (UERN), 2013. Revista Brasileira de Educação Médica, 39, 32-40.

Cascella, M., Rajnik, M., Aleem, A., Dulebohn, S., \& Di Napoli, R. (2021). Features, evaluation, and treatment of coronavirus (COVID-19). StatPearls.

do Rego, R. M., Marques, N. A., da Costa Monteiro, P., de Oliveira, C. L. B., de Almeida Lins, N. A., \& Caldas, C. A. M. (2019). O perfil atual do estudante de Medicina e sua repercussão na vivência do curso. Pará Research Medical Journal, 2(1-4), 0-0.

de Sousa Furtado, E., de Oliveira Falcone, E. M., \& Clark, C. (2003). Avaliação do estresse e das habilidades sociais na experiência acadêmica de estudantes de medicina de uma universidade do Rio de Janeiro. Interação em Psicologia, 7(2).

de Sousa Neto, A. R., \& de Freitas, D. R. J. (2020). Utilização de máscaras: indicações de uso e manejo durante a pandemia da covid-19. Cogitare Enfermagem, 25.

Estrela, C. (2018). Metodologia Científica: ciência, ensino, pesquisa. Editora Artes Médicas.

Freitas, C. A. D., Arruda, G. F. A. D., Arruda, G. C. F. A. D., \& Feitosa, S. F. (2021). Estudantes de Medicina no enfrentamento da pandemia da Covid-19 no Brasil: reflexões éticas. Revista Brasileira de Educação Médica, 45.

Kaluf, I. D. O., Sousa, S. G. O., Luz, S., \& Cesario, R. R. (2019). Sentimentos do Estudante de Medicina quando em Contato com a Prática. Revista Brasileira de Educação Médica, 43, 13-22.

Lemos, K. M., Neves, N. M. B. C., Kuwano, A. Y., Tedesqui, G., Bitencourt, A. G. V., Neves, F. B. C. S., \& Lima, M. M. (2007). Uso de substâncias psicoativas entre estudantes de Medicina de Salvador (BA). Archives of Clinical Psychiatry (São Paulo), 34, 118-124.

Lima, R. L. D., Soares, M. E. C., Prado, S. N. D., \& Albuquerque, G. S. C. D. (2016). Estresse do estudante de medicina e rendimento acadêmico. Revista Brasileira de Educação Médica, 40, 678-684.

Loureiro, E. M. F. (2006). Estudo da relação entre o stress e os estilos de vida nos estudantes de Medicina (Doctoral dissertation).

Marin, G. A., de Araujo Caetano, I. R., Bianchin, J. M., \& Cavicchioli, F. L. (2021). Depressão e efeitos da COVID-19 em universitários. InterAmerican Journal of Medicine and Health, 4. 
Moreira, S. D. N. T., Vasconcellos, R. L. D. S. S., \& Heath, N. (2015). Estresse na formação médica: como lidar com essa realidade? Revista Brasileira de Educação Médica, 39, 558-564.

Ministério da Saúde. Protocolo de Manejo Clínico do Coronavírus (COVID-19) na atenção primária à saúde. 2020a. https://www.saude.gov.br/images/pdf/2020/marco/20/20200318-ProtocoloManejo-ver002.pdf.

Ministério da Saúde. Doença pelo coronavírus 2019. Ampliação da Vigilância, medidas não farmacológicas e descentralização do diagnóstico laboratorial. Ministério da Saúde: Secretaria de Vigilância em Saúde, 2020b. http://maismedicos.gov.br/images/PDF/2020_03_13_Boletim-Epidemiologico-05.pdf.

Neto, J. A. C., Sirimarco, M. T., de Almeida Delgado, A. A., Moutinho, B. D., Lara, C. M., \& Lima, W. G. (2013). Estudantes de medicina sabem cuidar da própria saúde? HU Revista, 39 (1 e 2).

Organização Pan Americana de Saúde - OPAS. 10 principais causas de morte no mundo, 2018 . <https://www.paho.org/bra/index.php?option=com_content\&view=article\&id=5638:10-principais-causas-de-morte-no-mundo\&Itemid=0>.

Ornell, F. E. L. I. P. E., Schuch, J. B., Sordi, A. O., \& Kessler, F. H. (2020). Pandemia de medo e COVID-19: impacto na saúde mental e possíveis estratégicas. Revista debates in psychiatry, 2020.

Rodriguez-Morales, A. J., Gallego, V., Escalera-Antezana, J. P., Méndez, C. A., Zambrano, L. I., Franco-Paredes, C., \& Cimerman, S. (2020). COVID-19 in Latin America: The implications of the first confirmed case in Brazil. Travel medicine and infectious disease, 35, 101613.

Sacramento, B. O., Anjos, T. L. D., Barbosa, A. G. L., Tavares, C. F., \& Dias, J. P. (2021). Sintomas de ansiedade e depressão entre estudantes de medicina: estudo de prevalência e fatores associados. Revista Brasileira de Educação Médica, 45.

Santa, N. D., \& Cantilino, A. (2016). Suicídio entre médicos e estudantes de medicina: revisão de literatura. Revista Brasileira de Educação Médica, 40, 772780.

Soeiro, A. C. V., Limonge, L. G., Lopes, N. S., \& Fayal, S. P. (2021). Abordagem do suicídio na educação médica: analisando o tema na perspectiva dos acadêmicos de medicina. Revista Brasileira de Educação Médica, 45.

Souza, P. G. A. D., Pôrto, A. C. C. D. A., Souza, A. D., Silva, A. G. D., \& Borges, F. T. (2020). Perfil socioeconômico e racial de estudantes de medicina em uma universidade pública do Rio de Janeiro. Revista Brasileira de Educação Médica, 44

Tavares, G. H., de Oliveira, D. P., Rodrigues, L. R., da Mota, C. G., de Sousa, T. F., \& Polo, M. C. E. (2020). Inatividade física no lazer durante a pandemia da COVID-19 em universitários de Minas Gerais. Revista Brasileira de Atividade Física \& Saúde, 25, 1-7.

Trindade, S. C., Sousa, L. F. F. D., \& Carreira, L. B. (2021). Generalized anxiety disorder and prevalence of suicide risk among medical students. Revista Brasileira de Educação Médica, 45.

Veras, R. M., Fernandez, C. C., Feitosa, C. C. M., \& Fernandes, S. (2020). Perfil socioeconômico e expectativa de carreira dos estudantes de Medicina da Universidade Federal da Bahia. Revista Brasileira de Educação Médica, 44.

Webster, R. K., Brooks, S. K., Smith, L. E., Woodland, L., Wessely, S., \& Rubin, G. J. (2020). How to improve adherence with quarantine: rapid review of the evidence. Public Health, 182, 163-169.

World Health Organization. (1998). The World Health Report 1998: Life in the 21st century a vision for all. In The world health report 1998: life in the 21st century A vision for all (pp. 241-241).

World Health Organization. (2020). Noncommunicable diseases: Progress monitor 2020. <http://www.who.int/topics/chronicdiseases/en/〉.

Zonta, R., Robles, A. C. C., \& Grosseman, S. (2006). Estratégias de enfrentamento do estresse desenvolvidas por estudantes de medicina da Universidade Federal de Santa Catarina. Revista Brasileira de Educação Médica, 30, 147-153. 